\title{
Some Results on Many-Body Scattering Theory
}

\author{
PAut Fforkbush* \\ Department of Mathematics, University of Michigan, Ann Arbor, Michigan
}

\begin{abstract}
Several problems are considered related to the study of the $T$-matrix in the manybody scattering situation. Expanding previous results, the $T$-matrix is studied outside the realm of perturbation theory, with relation to establishing its analytic properties under the rotation of the final momenta from real to complex values, $\mathbf{k} \rightarrow(1+\theta) \mathbf{k}$. The geometry of the contour distortion is expressed so as to involve nonperturbative $T$ matrix element subblocks with incoming and outgoing momenta uniformly rotated. An unrelated elementary Hilbert space argument is given to bound the contributions of high-energy intermediate states to the resolvent identity.
\end{abstract}

\section{INTRODUCTION}

The present paper considers question arising in studying the $T$-matrix in manybody scattering theory. One goal is to develop a proof of the asymptotic completeness property, for certain types of potentials; and it is hoped that the results of this paper will be useful to this end. Asymptotic completeness has been established by Faddeev for scattering with three or less bodies (l), by studying the resolvent identity. The difficult proof he developed employed many spaces of Holder continuous function, the establishment of compactness for certain operators on these spaces being the crux of the proof. The questions studied in this work relate to a procedure slightly different from that of Faddeev. K. Hepp, has recently announced a proof of asymptotic completeness in the general situation, by a still different method (4).

It is natural from the experience in much of recent physics to try to exploit analyticity properties wherever possible. Asymptotic completeness has been established by studying the resolvent identity in the limit $\epsilon \rightarrow 0$. Basically then one must know properties of the $T$-matrix in the limit $\varepsilon \rightarrow 0$. In this limit equations for the $T$-matrix, such as Weinberg's equations (2) become singular in Hilbert space. However if the momenta were all to be rotated the equations are perfectly regular as $\varepsilon \rightarrow 0$. The natural idea is to try to solve the equations for the $T$-matrix at a rotated angle and then to rotate back to the real axis. In a previous paper [Reference (3), henceforth referred to as SAN] it was shown that such a rotation is possible for suitable potentials in perturbation theory. (In fact, the behavior at

* Sloan Foundation Fellow. 
infinity of the integrals was not studied.) The present paper extends the results of SAN in several directions.

By iterating the equations for the $T$-matrix, the $T$-matrix may be expressed, in many ways, as a sum of perturbation-like terms. Each term is canonically associated to a diagram differing from a perturbation theory diagram only in that it may contain blocks representing nonperturbative quantities. To the diagram corresponds an integral, the integration over internal momenta, in which distortions of the integration contour will be considered. Remaining this vague for the moment, we will study the question in this paper of rotating the final momenta in such a generalized diagram keeping the initial momenta real. For each subblock of the diagram its incoming and outgoing momenta will be at a constant angle, the same for all subblocks. (As seen in Section I, the momenta are actually on a surface that for small momenta corresponds to a rotation, but for which the imaginary part of the momenta remains bounded.) Thus the $T$-matrix with initial momenta real and final momenta rotated is expressed in terms of nonperturbative quantities-all evaluated on rotated contours where life should be relatively easy. The angle of rotation can be picked non zero at threshholds, a fact not established in SAN even in perturbation theory. The integration contour can be kept constant as the energy approaches the real axis, for small intervals. In (5) a special case of the present program is generalized to the relativistic situation.

In Section I the idea of a rotated contour is generalized so that the imaginary parts of the momenta all remain bounded. Basically the momenta are rotated for small $\mathbf{k}$ but flattened back for large $\mathbf{k}$. However it is not quite that simple.

Section II describes the required expression of the $T$-matrix as a sum of perturbation-like terms.

Section III establishes the integration contour over internal momenta for the situation described above, including a brief consideration of its smoothness.

Section IV contains a Hilbert space estimate bounding high-energy contributions to the resolvent identity, valid as $\varepsilon \rightarrow 0$. This appears to a useful theoretical result.

Not studied in this paper is the behavior of the nonperturbative subblocks on the rotated contours. We assume they are integral operators behaving well enough at infinity to allow the contour distortions at infinity, and are sufficiently analytic to allow the analytic continuation. Such properties are expected ones; bound states are allowed for, including bound states at positive energy, a possibility so far only excluded in the two body case. For the momenta rotated by a suitably small angle, the energy must be continuable onto part of the second sheet, up to some angle less than twice the contour rotation. The specific study of these subblock properties is not here undertaken. In actuality the results of the present paper are in some sense elementary, the results of the first three sections are trivial for the two body system. The fact that they are not long in the literature is a reflection of past lack of attention to many-body scattering theory. 


\section{CORRECTING ROTATED CONTOURS AT INFINITY}

In the two-body problem the problem of this section is trivial. One selects a contour with $\operatorname{Im} \mathbf{k}=c \operatorname{Re} \mathbf{k}$ for small $\mathbf{k}$ and smoothly curved down to $\operatorname{Im} \mathbf{k}=\mathbf{0}$ for large $\mathbf{k}$. In defining a contour in general we will do so by finding a map from $E^{N}$ to $E^{N}$, the map from the real part of $\mathbf{k}$ to the imaginary part of $\mathbf{k}$. We will use the notation of SAN, involving preferred directions. We are faced with a purely geometrical problem. In this problem, whose precise statement will follow, the second condition is the condition that is both trivial and unnecessary in the two-body case. It is very important to be consistent with the work of Section III, and the here untreated problem of solving integral equations for the $T$-matrix (our nonperturbative blocks) on rotated contours.

\section{Geometrical Problem}

There are given $\mathbf{n}_{1}, \ldots, \mathbf{n}_{s}, s$ distinct unit vectors in $E^{N}$, the preferred directions. It is desired to construct a map $\rho$, from $E^{N}$ to itself with the following three properties:

(1) $f$ is the identity map on the unit ball in $E^{N}$.

(2) If $J$ is any subset of the integers $1, \ldots, s$, and

$$
\mathbf{r}=\mathbf{V}+\sum_{i \in J} a_{i} \mathbf{n}_{i} \quad \text { with } \quad|\mathbf{V}|<1
$$

(each point in $E^{N}$ is identified in the standard way with a vector connecting it to the origin) then

$$
f(\mathbf{r})=\mathbf{V}+\sum_{i \in J} b_{i} \mathbf{n}_{i}
$$

for some set of $b_{i}$ 's.

(3) $f$ is $c^{\infty}$, bounded, and each derivative is uniformly bounded. That is $f$ is bounded and uniformly smooth over all space.

\section{SOLUTION}

The solution we give is quite explicit. We introduce the notation, $H_{J}$, for the subspace in $E^{N}$ (containing the origin) spanned by the subset $\left\{\mathbf{n}_{i} \mid i \in J\right\}$, one subspace for each subset $J$ of the integers $1, \ldots, s$. For each point $x \in E^{N}$ we distinguish two possibilities:

(a) if the Euclidean distance from all the $H_{J}$ 's is greater than 5 , we associate to $x$ the sphere of radius 3 about $x, u_{x}$. On $u_{x}$ we define the map, $f_{x}$, carrying all of $u_{x}$ to the origin in $E^{N}$. 
(b) Let the set

$$
H_{J_{\alpha_{1}}, \ldots, H_{J_{\alpha_{t}}}}
$$

consist of exactly the $H_{J}$ 's whose distance from $x$ is less than or equal to 5 . Let $W_{x}$ be the subspace given by the intersection of these $H_{J}$ 's. To such an $x$ we associate the sphere of radius 5 centered at $x, u_{x}$, and the map $f_{x}$ carrying each point in $u_{x}$ onto the perpendicular subspace to $W_{x}$ passing through the origin, by perpendicular projection.

Now we pick a rectangular mesh in $E^{N}$ with mesh size in each direction of length $1 / 4 N$. To each point $p$ of the mesh we associate a point $x(p)$ with the property that the sphere of radius $5 / 4$ about $p$ is contained in $u_{x(p)}$. Let $\phi_{1}(x), \phi_{2}(x)$ be $c^{\infty}$ functions on $E^{N}$ with

$$
\begin{aligned}
\phi_{1}(x)=1 & |x| \leqslant 2, \\
\phi_{2}(x)=1 & |x| \leqslant 4, \\
\phi_{1}(x)=0 & |x| \geqslant 3, \\
\phi_{2}(x)=0 & |x| \geqslant 5, \\
0 \leqslant \phi_{1}(x) \leqslant 1, & 0 \leqslant \phi_{2}(x) \leqslant 1 .
\end{aligned}
$$

Finally we can write a choice for $f(x)$ :

$$
f(y)=\frac{\sum_{p} \phi_{\alpha(p)}(y-x(p)) f_{x(p)}(y)}{\sum_{p} \phi_{\alpha(p)}(y-x(p))}
$$

$\alpha(p)=1$ or 2 according to whether $u_{x(p)}$ is a sphere of radius 3 or 5 . (The functions $f_{x}$ are naturally extended, it matters not how, to all of $E^{N}$. The additions and and multiplications take place as vector operations in $E^{N}$.)

\section{II. $M$-NICE DECOMPOSITIONS OF THE RESOLVENT}

In this section we propose to describe expressions of the resolvent $1 /(E-H)$ as finite sums of terms, $R_{i}$, the partial resolvents, with certain useful properties.

$$
1 /(E-H)=\sum R_{i}
$$

A given decomposition such as we consider will be $M$-nice for some integer $M$ if each $R_{i}$ in its decomposition is $M$-nice. A decomposition $M$-nice will also be $N$-nice for any $N<M$, and in particular the decompositions are not unique.

First, to get some notation settled we consider

$$
H=H_{0}+\sum_{i<j} V_{i j} \quad i, j \leqslant n
$$


To every partition $I$, of the first $n$ integers we define $V_{I}$ the subsum of the sum $\sum_{i<j} V_{i j}$ consisting only of interactions between pairs of particles that lie in the same subset of the partition. Corresponding to $H_{I}=H_{0}+V_{I}$ we can expand $1 /\left(E-H_{I}\right)$ in perturbation theory (treating $V_{I}$ as a perturbation) each term corresponding to a diagram. A diagram is called $I$-laced if any two particles in the same subset of the partition $I$ are connected by a sequence of interactions, and no two particles in different subsets. A diagram is $S$-connected $I$-laced if it can be realized as a sequence of $S I$-laced diagrams.

The decompositions are obtained by a finite sequence of applications of three types of operations,

(1) Use of the two identities

$$
\begin{aligned}
\frac{1}{E-H_{0}-V_{l}} & =\frac{1}{E-H_{0}-V_{J}}+\frac{1}{E-H_{0}-V_{J}}\left(V_{I}-V_{J}\right) \frac{1}{E-H_{0}-V_{I}} \\
& =\frac{1}{E-H_{0}-V_{J}}+\frac{1}{E-H_{0}-V_{I}}\left(V_{I}-V_{J}\right) \frac{1}{E-H_{0}-V_{J}}
\end{aligned}
$$

with $J$ a refinement of $I$.

(2) Writing $V_{I}-V_{J}$ as a sum of individual potentials to obtain a sum of terms.

(3) Recombination of terms obtained.

It is understood that the identities in (1) may be used for any denominator appearing anywhere in a given expression.

A partial resolvent is thus a finite sum of terms of the type

$$
\frac{1}{E-H_{0}-V_{I \alpha_{1}}} V_{\beta_{1} \gamma_{1}} \frac{1}{E-H_{0}-V_{I \alpha_{2}}} V_{\beta_{2} \gamma_{2}} \cdots
$$

It is now necessary to define a perturbation-like diagram. A perturbation diagram is defined by a finite sequence of pairs of elements, $\left(\beta_{1} \gamma_{1}\right),\left(\beta_{2} \gamma_{2}\right), \ldots$, the pairs corresponding to the sequence of interactions $V_{\beta_{1} \gamma_{1}}, V_{\beta_{2} \gamma_{2}}, \ldots$, and the perturbation term itself

$$
\frac{1}{E-H_{0}} V_{\beta_{1} \gamma_{1}} \frac{1}{E-H_{0}} V_{B_{2} \gamma_{2}} \cdots
$$

A perturbation-like diagram is a finite sequence each element of which is either a partition of the integers, $1,2, \ldots, n$, or a pair of these integers. For example for $n=3$ a perturbation-like diagram of length 4 is

$$
[(1,2)] \cdot[(1,2,3)] \cdot[(1,2)(3)] \cdot[(2,3)]
$$


A perturbation diagram is a perturbation-like diagram in which only pairs appear. A perturbation-like diagram of length $s$ is representable as a product

$$
G_{1} G_{2}, \ldots, G_{s}
$$

each $G_{i}$ pair or a partition. The partial resolvent $R$ will be said to be associated to this diagram provided

$$
R=L_{1} L_{2} \cdots L_{s} \cdot \frac{1}{E-H_{0}}
$$

with $L_{k}=\left[1 /\left(E-H_{0}\right)\right] V_{i j}$ if $G_{k}=(i, j)$ and if $G_{k}$ is the partition $I, L_{k}$ is a sum of terms of the form (2.1), with last denominator term omitted, such that the perturbation expansion of $L_{k}$ generates only $I$-laced diagrams.

The perturbation-like diagram represented as $G_{1} G_{2}, \ldots, G_{s}$ is $M$-nice if the following property holds:

Property. If $G_{k}$ is the partition $I$ there is an $r \geqslant 1$ and a $t \leqslant s$ such that in the perturbation-like diagram

$$
G_{r} \cdot G_{r+1}, \ldots, G_{k}, \ldots, G_{t-1} G_{t}
$$

(1) each $G_{i}$ is either a pair of particles in the same subset of $I$, or a partition that is a refinement if $i \neq k$.

(2) Any perturbation diagram dominated by $G_{r} \cdot G_{r+1}, \ldots, G_{k-1}$ or by $G_{k+1} \cdot G_{k+2}, \ldots, G_{t}$ is $M$-connected $I$-laced, where here a perturbation diagram is dominated by a perturbation-like diagram provided it is expressible as a sequence of diagrams, each associated to one of the $G$ 's in proper sequence, the diagram corresponding to $G_{i}$ being $(j, r)$ if $G_{i}$ is $(j, r)$ and being $J$-laced if $G_{i}$ is the partition $J$.

THEOREM. For any integer $M$, there is a set of partial resolvents $R_{1}, R_{2}, \ldots, R_{s}$ such that

(1) $1 /(E-H)=\sum R_{i}$

(2) Each $R_{i}$ is associated to an $M$-nice perturbation-like diagram. ( $R_{i}$ is M-nice.)

A proof of this is easily developed by ensuring this property for partitions of increasing refinement successively.

\section{THE GEOMETRY OF CONTOUR ROTATION}

In this section, the heart of the paper, the purpose is to define a contour of integration for each of the partial resolvents, corresponding to a suitable $M$-niceness to be specified, described in the last section. If the overall connectivity is great 
enough the final momenta are rotated into the complex plane, if not both initial and final momenta are real, in both cases all nonperturbative subblocks are uniformly rotated. The contour of integration will be specified by assigning to each set of real momenta values, a specification of imaginary values of the momenta. Thus the contour projects one to one onto its real values. As in SAN, upon which this section heavily relies, we begin with a geometrical problem.

\section{Basic Geometrical Problem}

There is in $E^{N}$ a finite set of unit vectors (the preferred directions) given. There is also given a finite set of spheres centered at the origin in $E^{N}$. The basic result is then that there is a $\delta$ and an integer $M$ such that if $x_{1}, x_{2}, \ldots, x_{s}$ is a sequence of vectors in $E^{N}$ with $x_{i+1}-x_{i}$ parallel to one of the preferred directions for every $i$, and if this set of vectors is $M$-connected, then there is a second set of vectors $y_{1}, \ldots, y_{s}$ with

(1) $y_{1}=0$

(2) $y_{s}=x_{s}$

(3) $y_{i+1}-y_{i} / / x_{i+1}-x_{i}$

(4) $y_{i} \cdot x_{i} \geqslant 0$ if $\left|x_{i}\right|$ is within $\delta$ of the radius of any one of the prescribed spheres.

\section{SKeTCH OF SOLUTION}

For a single sphere this problem has been solved in SAN. We use this solution to inductively solve the generalized problem. Arranging the spheres in order of decreasing radius,

$$
r_{1}, r_{2}, \ldots, r_{t},
$$

we proceed as follows. If the problem can be solved for the single sphere of radius $r_{1}$ for the problem $x_{1}, x_{2}, \ldots, x_{w}, w \leqslant t$ with $\left|x_{i}\right| \geqslant r_{2}+\delta_{2}, i \leqslant w$ then the present problem is solved by using this solution and $y_{i}=x_{i}, i>w$ (the $\delta_{i}$ is associated to $r_{i}$ as the $\delta$ of SAN, and $\delta_{i}$ can be picked so that $\delta_{i}=\lambda r_{i}$ for some $\lambda$ and all $r_{i}$.) By induction, if the problem can be solved for $r_{1}, \ldots, r_{k}$ and $x_{1}, x_{2}, \ldots, x_{w}$ with $\left|x_{i}\right| \geqslant r_{k+1}+\delta_{k+1}, i \leqslant w$ then the present problem is solved by using this solution and setting $y_{i}=x_{i}, i>w$. If this is not possible so that $\left|x_{w+1}\right|<r_{k+1}+\delta_{k+1}$ try to solve using Lemma 7 and Lemma 5 of SAN. This induction process solves the problem, it is easy to see that some $M$ suffices as each procedure utilized is ended after some connectivity sequence. In fact $M$ can be picked dependent only on the number of spheres and the preferred directions. 


\section{MODIFICATIONS}

Although we solved the basic problem above, we find it necessary to modify the geometrical solution above, to insure that the rotation angle can be picked finite at threshholds, that the final contour we get is smooth, that the imaginary parts of the momenta be bounded, and that the nonperturbative blocks have their external momenta uniformly rotated. We describe the modifications with varying degrees of completeness.

(1) We first treat the special case when the solution above proceeds via lemma 4 of SAN, i.e., the problem can be solved before any $x_{i}$ gets inside any of the spheres. Rather than use the procedure of lemma 4 we modify Lemma 4 of SAN as follows.

Let $x_{1}, x_{2}, \ldots, x_{w}$ have differences that pass through a spanning set. We choose $y_{1}, y_{2}, \ldots, y_{w}$ as follows.

Assume $x_{\alpha_{i}}-x_{\alpha_{i}-1}$ is a basis $i=1, \ldots, N$. Now let $x_{r} 1 \leqslant r \leqslant w$ be the $x$ with minimal length, $|x|$. Expand

$$
x_{r}=\sum c_{k t}\left(x_{\alpha_{k}}-x_{\alpha_{k}-1}\right)
$$

The solution for $y_{i}$ now is displayed.

$$
\begin{aligned}
& y_{1}=0, \\
& \begin{aligned}
y_{i}=\theta(i, r)\left(x_{i}-x_{r}\right) & -\sum_{k} c_{k}\left(x_{\alpha_{k}}-x_{\alpha_{k}-1}\right) \theta\left(i, \alpha_{k}\right), \\
\theta(\alpha, \beta) & =0 \quad \alpha<\beta \\
& =1 \quad \alpha \geqslant \beta .
\end{aligned}
\end{aligned}
$$

This modification guarantees in the perturbation theory case of SAN that the rotation can be picked finite at threshhold. The remaining modifications are more technical.

(2) The second modification is concerned with the innermost sphere. We imagine a sphere $1 / s$ ( $s$ a fixed integer) way between the intermost sphere and the next largest one. The procedure above is unmodified if the problem is solved $\left(x_{i}=y_{i}\right)$ before $x_{i}$ has magnitude less than the intermediate sphere radius we have imagined. If this is not the case, $y_{i}$ is kept zero until $x_{i}$ falls inside this sphere and thereafter lemmas 5 and 7 of SAN, or modification (1) is followed according to remaining possibilities. (The outer spheres are now irrelevant.) $s$ is picked to insure the dotproduct condition, this is no problem.

(3) For some large number $\lambda$ we consider the following possibility, three vectors $x_{a}, x_{b}, x_{c}$ appear in the same order, not necessarily consecutively, $x_{a}$ and $x_{c}$ 
are both less than $\lambda_{1}$ times the imaginated radius of (2) ( $\lambda_{1}$ a small number) and $\left|x_{b}\right|>\lambda \operatorname{Max}\left(\left|x_{a}\right|,\left|x_{c}\right|\right)$. If $\lambda$ is picked large enough one can modify the solution of (2) such that for every such triple of $x$ 's, $\left|y_{b}-x_{b}\right|<\lambda_{2} \operatorname{Min}\left(\left|x_{a}\right|,\left|x_{c}\right|\right)$. $\lambda$ and $\lambda_{1}$ must be chosen to satisfy certain simple conditions, to maintain the dot product condition. This modification is easily carried out, leaving $y_{a}$ and $y_{b}$ unaffected, leaving all $y_{i}$ corresponding to small $x_{i}$ alone and favoring small $\left|x_{i}-y_{i}\right|$.

(4) As in SAN, one can modify the procedure to keep the imaginary part of the momenta bounded. For this adjustment we put the diagrams external momenta on the type of contour established in Section I, if the external momenta are rotated. This modification described in SAN can be performed without changing the imaginary parts of the momenta, the $y$ 's corresponding to $x$ 's inside some fixed sphere.

\section{ESTABLISHMENT OF THE CONTOUR}

The $N$-dimensional space we have been dealing with is associated to the 3(n-1)-dimensional space of normalized momenta, with $H_{0}=\sum k^{2}$. The spheres of this section are at radii $E_{0}-\sum_{i \in J} E_{B_{i}}$, where $E_{B_{i}}$ are bound state energies of proper subsets of the particles (excepted to be negative) and the index set $J$ run through possible scattering channels. $E_{0}$ is the total c.m. energy at which one is interested in approaching the real axis. We remember that the integration contour can be picked independent of $E$ for small variations in $E$.

First we find a decomposition of the resolvent $M$-nice where $M$ is greater than the connectivity required to solve any geometrical problem faced. This depends only on the numbers of bound states in different channels and the masses of all the particles. To a given partial resolvent we associate a perturbation diagram as follows. Pick an arbitrary perturbation-like diagram to which the partial resolvent is associated. To this diagram associate a perturbation diagram by a associating to each pair in the perturbation diagram, and to each $I$-laced nonperturbative block in one diagram an arbitrary 2-connected I-laced perturbation diagram, with minimal number of interactions. The middle intermediate state in each block is picked to have real and imaginary parts of its momentum vector of minimum length allowed by the external momenta. The contour of this perturbation diagram yields the contour of the perturbation-like diagram, or partial resolvent, by omitting the behavior of the artifically introduced momenta in each block. We omit consideration of modifying the contour to guarantee that the external momenta in each subblock lie on a contour established in Section I. The procedure is relatively clear.

To establish a full contour one must mold the various programs for solving the geometrical problem together using a $c^{\infty}$ partition of unity as in SAN. One observes 
that each individual program that works over some range of internal momenta can be expressed by a linear relation between the $x$ 's and $y$ 's. Some of the programs must be applied outside their initial realm, for example, in modification (1) the "smallest momentum" selected may not be the smallest. There are always overlaps of acceptable programs except at threshholds. It is relatively easy to show there is a contour $c^{\infty}$ except at threshholds. We claim in fact it can be constructed Holder continuous for some index. If this fact or more detailed properties are used in the future, a fuller discussion may be presented.

\section{A BOUND ON HIGH-ENERGY CONTRIBUTIONS TO THE RESOLVENT IDENTITY}

We put ourselves in a very general perturbation theory setting, $H_{0}$ is a positive self adjoint operator in Hilbert space. $V$ is a self adjoint bounded operator.

$$
|V|<c
$$

We let $H=H_{0}+V$. We write the spectral resolution of $H_{0}$,

$$
H_{0}=\int \lambda d E_{\lambda}
$$

and define

$$
P_{\lambda_{0}}=\int_{\lambda_{0}}^{\infty} d E_{\lambda}
$$

Starting with the resolvent identity.

$$
\frac{1}{E-H+i \varepsilon}-\frac{1}{E-H-i \varepsilon}=(-2 i \varepsilon) \frac{1}{E-H+i \varepsilon} \cdot \frac{1}{E-H-i \varepsilon}
$$

with $E$ real and $1>\varepsilon>0$. We integrate $E$ from $a$ to $b$.

$\int_{a}^{b}\left[\frac{1}{E-H+i \varepsilon}-\frac{1}{E-H-i \varepsilon}\right] d E=\int_{a}^{b}(-2 i \varepsilon) \frac{1}{E-H+i \varepsilon} \cdot \frac{1}{E-H-i \varepsilon} d E$

This equation is of prime importance in the study of the asymptotic completeness relation, particularly in the limit $\varepsilon \rightarrow 0$.

We insert $P_{\lambda_{0}}$ in the right-side of (4.2), making the definition

$$
\int_{a}^{b}(-2 i \varepsilon) \frac{1}{E-H+i \varepsilon} P_{\lambda_{0}} \frac{1}{E-H-i \varepsilon} d E=O\left(a, b, \varepsilon, \lambda_{0}\right) .
$$

letting $\Delta=\lambda_{0}-b>0$, the result we are after is the following: 
Theorem. $\left|O\left(a, b, \varepsilon, \lambda_{0}\right)\right|<k / \Delta^{2}$ with $k$ dependent only on $a, b, V$, and $H_{0}$, not on $\varepsilon$.

Proof. Using well known identities we write

$$
\begin{aligned}
O\left(a, b, \varepsilon, \lambda_{0}\right)= & \int_{a}^{b} d E(-2 i \varepsilon)\left[\frac{1}{E-H_{0}+i \varepsilon}+\frac{1}{E-H+i \varepsilon} V \frac{1}{E-H_{0}+i \varepsilon}\right] \\
& \cdot P_{\lambda_{0}} \cdot\left[\frac{1}{E-H_{0}-i \varepsilon}+\frac{1}{E-H_{0}-i \varepsilon} V \frac{1}{E-H-i \varepsilon}\right]
\end{aligned}
$$

We estimate each of the three types of terms (two of the four terms are related by symmetry) in expanding this equation with easy estimates.

$$
\begin{aligned}
& \left|\int_{a}^{b} d E(-2 i \varepsilon) \frac{1}{E-H_{0}+i \varepsilon} P_{\lambda_{0}} \frac{1}{E-H_{0}-i \varepsilon}\right| \\
& \quad \leqslant\left|(b-a)(-2 i \varepsilon) \frac{1}{\Delta^{2}}\right| \leqslant \frac{c_{1} \varepsilon}{\Delta^{2}} \\
& \left|\int_{a}^{b} d E(-2 i \varepsilon) \frac{1}{E-H_{0}+i \varepsilon} P_{\lambda_{0}} \frac{1}{E-H_{0}-i \varepsilon} V \frac{1}{E-H-i \varepsilon}\right| \\
& \quad \leqslant\left|(b \quad a)(-2 i \varepsilon) \frac{1}{\Delta^{2}} c \frac{1}{\varepsilon}\right| \leqslant \frac{c_{2}}{\Delta^{2}} \\
& \left|\int_{a}^{b} d E(-2 i \varepsilon) \frac{1}{E-H+i \varepsilon} V \frac{1}{E-H_{0}+i \varepsilon} P_{\lambda_{0}} \frac{1}{E-H_{0}-i \varepsilon} V \frac{1}{E-H-i \varepsilon}\right| \\
& \quad \leqslant\left|\int_{a}^{b} d E(-2 i \varepsilon) \frac{1}{E-H+i \varepsilon} V \frac{1}{\Delta^{2}} V \frac{1}{E-H-i \varepsilon}\right| \\
& \quad \leqslant\left|(2 \varepsilon) \frac{1}{\Delta^{2}} c^{2} \int_{a}^{b} d E \frac{1}{E-H+i \varepsilon} \cdot \frac{1}{E-H-i \varepsilon}\right| \\
& \quad \leqslant\left|(2 \varepsilon) \frac{1}{\Delta^{2}} c^{2} \int_{-\infty}^{\infty} d E \frac{1}{E-H+i \varepsilon} \cdot \frac{1}{E-H-i \varepsilon}\right| \\
& \quad \leqslant\left|\pi \frac{1}{\Delta^{2}} c^{2}\right| \leqslant \frac{c_{3}}{\Delta^{2}} .
\end{aligned}
$$

This completes the proof.

\section{v. OUTLOOK}

The key remaining problem is the study of the nonperturbative blocks on the rotated contours of Section I. If these can be established as well behaved operators in certain spaces of functions then their insertion in the full expressions for the 
T-matrix in Section III should not be difficult. Our present prejudice is to work with functions uniformly Holder continuous in all variables, and uniformly bounded.

\section{REFERENCES}

I. L. D. FADDEEv, Mathematical Problems of the Quantum Theory of Scattering for a Three Particle System, AERE-Trans 1002, United Kingdom Atomic Energy Authority Translation.

2. Steven Weinberg, Phys. Rev. 133, B232 (1964).

3. P. G. FeDERBush, Journal of Mathematical Physics, 8, 2415 (1967).

4. K. HEPP, to be published.

5. G. D. MCCARTOR AND J. NutTall, to be published. 